\title{
The Ketogenic Diet as a Potential Therapy in Down syndrome
}

\author{
Kyle N. Kaneko' ${ }^{1 *}$, Miki Wong ${ }^{1,3}$, Michael J. Corley ${ }^{1,2}$, Ryan W.Y. Lee ${ }^{1,2,3}$ \\ 'Shriners Hospitals for Children ${ }^{\circledR}$-Honolulu, Department of Research, Honolulu, HI, USA \\ ${ }^{2}$ John A. Burns School of Medicine, University of Hawaii School of Medicine, Honolulu, HI, USA \\ ${ }^{3}$ Milestones Center for Pediatric Neurodevelopment, Honolulu, HI, USA
}

\section{Article Info}

\section{Article Notes}

Received: March 03, 2018

Accepted: March 22, 2018

\section{${ }^{*}$ Correspondence:}

Mr. Kyle Kaneko

3438 Pinao Street, Honolulu, HI, 96822, USA;

Telephone: 808-227-7789;

E-Mail: kanekokyle067@yahoo.com

(c) 2018 Kaneko KN. This article is distributed under the terms of the Creative Commons Attribution 4.0 International License.

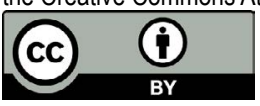

\section{Keywords}

Ketogenic diet

Down syndrome

Cognition

Behavior

Therapy

Treatment

Nutrition

\section{ABSTRACT}

Down syndrome is a common genetic intellectual disability seen in humans. Currently, therapeutic interventions are inadequate in improving the quality of life for individuals with Down syndrome that have cognitive and behavioral impairments. Nutrition therapies for Down syndrome have focused on addressing obesity but not intellectual disability and cognitive decline. The ketogenic diet is a very low carbohydrate, moderate protein, and high fat diet used to treat childhood and adult epilepsy, however, there is growing interest in its potential to improve cognition and behavior. There is evidence suggesting that the ketogenic diet may be effective in treating comorbidities associated with Down syndrome such as early onset of Alzheimer's disease and dementia. This review aims to discuss the ketogenic diet and the potential benefits that the diet may provide in neurodevelopmental and neurodegenerative diseases. We propose that the ketogenic diet may be a therapeutic option for cognitive decline in Down syndrome and warrants investigation.

\section{Introduction}

Down syndrome (DS), or trisomy 21, is a chromosomal disorder that is considered the most common genetic intellectual disability in humans, with a worldwide prevalence of 1 in 1100 live births ${ }^{1}$. DS is characterized by distinctive facial and skeletal features, hypotonia, developmental delay, and intellectual disability ${ }^{1}$. Common comorbidities include congenital heart disease, obesity, congenital ophthalmological disorders, epilepsy, hearing loss, and psychiatric disorders such as attention deficit-hyperactivity disorder and autism spectrum disorder (ASD) ${ }^{2}$.

Intellectual disability and behavioral problems are often the most impairing symptoms for individuals and families with DS. Approximately $80 \%$ of individuals with DS have moderate cognitive impairment ${ }^{3}$. Individuals with DS experience delays in cognitive development with specific deficits in speech, language production, verbal short-term memory, and explicit long term memory ${ }^{4}$. Cognitive impairment exists in the majority of individuals with DS, however, expressivity and severity vary considerably ${ }^{4}$.

The underlying mechanisms of cognitive dysfunction in DS is not well understood due to the wide variation of phenotypic expression in this disorder. In addition, individuals with DS experience an earlyonset cognitive decline characteristic of Alzheimer's disease (AD). $\mathrm{AD}$ affects $20-50 \%$ of DS individuals in their fifties, and $30-75 \%$ of 
those in their sixties ${ }^{5}$. Having 3 copies of chromosome 21 results in an overproduction of brain amyloid due to an over-expression of the amyloid precursor protein gene $e^{6}$. Nearly all show the same neuropathological changes such as senile plaques (SP-beta-amyloid protein) and neurofibrillary tangles (hyperphosphorylated tau protein) that meet criteria for a diagnosis of AD by the age of $40^{5}$. Higher plasma amyloid beta levels have been associated with poor communication skills, worse performance in a semantic verbal fluency task, and a higher Dementia Questionnaire for Persons with Intellectual Disability score $^{7}$.

Epilepsy, a common comorbidity in $\mathrm{DS}^{8}$, is also thought to contribute to cognitive dysfunction ${ }^{9}$. Recent prevalence data estimates that $12.4 \%$ of the DS population have a seizure disorder ${ }^{1}$. Cognitive symptoms of dementia in individuals with DS include forgetfulness, problems with learning, impaired short-term memory, and deficits in visuospatial organization ${ }^{2,4}$. McCarron et al. ${ }^{9}$ longitudinally followed 77 individuals diagnosed with DS and dementia and found that epilepsy was associated with dementia and sensory impairments in these individuals. Lott et al. ${ }^{10}$ looked at 53 adults with DS and AD-type dementia and demonstrated a positive correlation between seizure activity and the severity and duration of cognitive impairment compared to DS individuals without seizures. In addition, individuals with DS that develop late onset seizures (greater than 45 years of age) are significantly more likely to develop AD-like symptoms ${ }^{11}$. Frequent seizures may induce irregular sprouting of nerve fibers, reduced neurogenesis, and abnormal neurotransmitter function $^{12}$, which may contribute to cognitive decline.

Treatment for cognitive decline in DS remains a challenge. There is a lack of therapeutic interventions to improve the quality of life for those with DS. There is a lack of sufficient evidence for various pharmacological interventions including donepezil, memantine, simvastatin, acetyl-L-carnitine and other cholinesterase inhibitors regarding efficacy for treatment of cognitive decline in $\mathrm{DS}^{13}$. Furthermore, supplementation with antioxidant vitamins and minerals in children with and without folinic acid have shown little or no evidence in improving the psychomotor and language development in children with $\mathrm{DS}^{14}$. Nutritional therapies for DS have focused on obesity but fail to address intellectual disability and cognitive decline ${ }^{15}$.

The ketogenic diet (KD) is an established, effective treatment for patients with certain defects in glucose transporter $^{1}$, pyruvate dehydrogenase deficiencies, and intractable epilepsy ${ }^{16}$. The mechanisms behind its anticonvulsant effects have yet to be fully understood, however, there is growing evidence of the neuroprotective properties of the KD and ketone bodies in particular, and as such, increasing interest in using the KD to treat other neurological disorders. Recent studies have investigated cognition and behavioral changes in children with epilepsy on the $\mathrm{KD}^{17,18}$, autism spectrum disorder ${ }^{19,20,21}$, and developmental delay ${ }^{22}$. To our knowledge, there have not been any case studies demonstrating the effects of the KD in DS. This review aims to discuss the KD as potential therapy for treating cognitive dysfunction in individuals with DS.

\section{History of the Ketogenic Diet}

Fasting has been used as a treatment for seizures and other ailments for centuries. Hippocrates in $400 \mathrm{BC}$ described a man who was successfully rid of seizures with the complete abstinence from food and water. In 1921, Dr. Rawle Geyelin reported on seizure cessation in a young child as a result of fasting at the American Medical Association convention that year ${ }^{23}$. This child was under the care of Dr. Hugh Conklin, an osteopathic physician who had been treating epileptic seizures under a water only fasting protocol that included daily osteopathic treatments. Moreover, Geyelin reported that he had treated 26 of his own epileptic patients with fasting, and 18 showed a significant improvement ${ }^{23}$. One month later, Mayo Clinic physician Dr. Russell Wilder proposed the use of a high fat, low carbohydrate diet designed to induce the ketogenesis observed in starvation as an alternative and sustainable treatment to fasting for epileptic seizures. Dr. Mynie Peterman was the first to report designing and implementing such a diet in $1924^{23}$. The KD, as coined by Dr. Wilder, gained popularity as a treatment for epilepsy but fell into gradual disuse by the 1940s with the introduction of diphenylhydantoin and subsequent anti-epileptic drugs. However, there is a growing interest in the KD in recent years due to the resurgence of its use in the treatment of epilepsy ${ }^{24-27}$ and other neurologic and metabolic disorders ${ }^{28,29}$.

The KD is a high fat, moderate protein, and very low carbohydrate diet that induces the hepatic production of ketone bodies (beta-hydroxybutyrate (BHB) and aceteoacetate $)^{16}$. KDs mimic starvation and were developed for use in humans to treat epilepsy based on the long record of observations that fasting reduces seizures ${ }^{16}$. The reduction in dietary carbohydrates decreases the need for insulin production. This deactivates enzymes that stimulate glycogenesis and lipogenesis. Once glycogen reserves are sufficiently exhausted, fat is mobilized as an alternative fuel source to supply needed acetyl coenzyme A for energy. This leads to an over-production of ketone bodies acetoacetate and BHB, which are produced by the mitochondria in the liver. The primary ketone body produced is acetoacetate, while the primary circulating ketone body is BHB. BHB and acetoacetate cross the blood brain barrier and are converted to acetyl coenzyme $A$, which 
enters the Krebs cycle to produce adenosine triphosphate in the brain mitochondria ${ }^{16}$.

The mechanisms underlying the anti-convulsant effects of the KD are not fully understood, although hypotheses center on metabolic shifts that occur in a state of ketosis and neuroprotective properties of the ketone bodies themselves ${ }^{30}$. Clinical and animal research models affirm that ketosis is necessary for effectiveness, although the level of ketone production may not directly impact seizure control. For example, the Low Glycemic Index Treatment (LGIT) diet is used in the treatment of epilepsy ${ }^{31}$. Studies so far demonstrate a similar efficacy to the classic KD and the modified Atkins diet (MAD) ${ }^{32}$, yet patients on the LGIT tend to maintain a milder state of ketosis due to the more liberal allowance of low glycemic index carbohydrates. Rather than hypothesizing a singular mechanism, it is plausible that the diet exerts its effects through multiple mechanisms working together synergistically in response to glucose restriction and the resultant availability of ketone bodies ${ }^{33}$. One mechanism recently hypothesized by Giordano et al. ${ }^{34}$ involves neuroactive peptides such as ghrelin and insulin which are modulated by the KD. Insulin production is reduced on a very low carbohydrate, moderate protein diet. Insulin levels have been linked to seizure induction and ghrelin and leptin to seizure suppression. Lucchi et al. ${ }^{35}$ demonstrated that peroxisome proliferator-activated receptor gamma (PPAR $\gamma$ ) was able to modulate seizures through activation of a ghrelin receptor antagonist (EP-80317) ${ }^{34}$. Similarly, Simeone et al. ${ }^{36}$ found that mice on the KD increased nuclear expression of PPAR $\gamma$ in the brain and reduced the frequency of seizures. Mice given an antagonist of PPAR $\gamma$ had no KD-mediated changes in PPAR $\gamma^{2}$ expression and had no anti-seizure effects seen. These results suggest that PPAR $\gamma$ plays a role in the anticonvulsant properties seen in the $\mathrm{KD}^{36}$.

\section{Therapeutic Applications of the Ketogenic Diet}

\section{Epilepsy}

Epilepsy is a common co-morbidity in individuals with DS, with a reported prevalence of $13 \%{ }^{37}$. Epilepsy in childhood is often correlated with cognitive dysfunction and emotional and behavioral problems ${ }^{38,39}$. The KD has repeatedly been shown to improve seizure control in children with intractable epilepsy ${ }^{24-27,40,41}$. Recent studies have investigated the effect of the KD on cognition and behavior in children and adolescents with epilepsy. Pulsifer et al. ${ }^{17}$ conducted the first prospective study of the effects of the KD on development, behavior, and parenting stress. 65 children with intractable seizures, aged 18 months to 14 years 6 months were included in the study. The children were evaluated before KD initiation and 1 year after KD implementation. There was a significant reduction in seizure frequency, from 25 seizures per day at baseline, to a mean daily seizure frequency of 1.8 seizures per day after 1 -year follow up ${ }^{17}$. Children who continued the KD beyond 1 year showed a significant improvement in overall developmental functioning (as measured by the mean developmental quotient), with significant behavioral improvements in attention and social functioning ${ }^{17}$.

Hallbook et al. ${ }^{42}$ evaluated sleep structure during KD treatment in children with therapy-resistant epilepsy while correlating possible alterations with changes in clinical effects on seizure reduction, quality of life and behavior. 18 children were examined with polysomnographic recordings before and after 3 months of KD treatment. 11 children continued the KD and were also evaluated after 12 months of KD treatment. The KD was found to improve sleep quality, increased rapid-eye-movement sleep, which seemed to contribute to improvement in attentional behavior $^{42}$.

The first randomized controlled trial to evaluate the cognitive and behavioral effects of the KD was conducted by Ijff et al. ${ }^{18}$. A total of 50 patients were enrolled in the study, with 28 patients in the KD group and 22 in the control group. Follow up cognitive and behavioral assessments were performed approximately 4 months after KD initiation. Patients on the KD had significantly higher scores on the subscale 'vigor' (energy) than patients in the control group. There was a positive correlation with seizure reduction in the KD group; greater seizure reduction resulted in a greater vigor score. Patients on the KD also had significantly higher scores on the subscale 'productivity', were less anxious, and had less mooddisturbed behavior than the control group ${ }^{21}$. Treatment with the KD resulted in a positive impact on behavioral and cognitive functioning in children and adolescents with refractory epilepsy ${ }^{21}$.

The KD has been increasingly used to treat refractory epilepsy, however some evidence suggests that the KD may cause a paradoxical increase in epileptic activity. In a retrospective review of 21 patients treated with the KD and concurrent anti-epileptic medications, Lucchi et al. $^{43}$ reported 3 patients with worsening of epileptic seizures as reflected on the electroencephalogram and reported seizure frequency. In 1 of these patients, seizures transiently worsened in severity (with no change in frequency), with an overall seizure improvement of $78 \%$. Careful monitoring of this phenomenon should be done when KD intervention is used.

\section{Alzheimer's disease and Dementia}

Almost all adults with DS have neuropathological characteristics of $\mathrm{AD}$ such as amyloid plaques and neurofibrillary tangles by 40 years of age $\mathrm{e}^{5,44}$. Concentrations of brain amyloid increase over time and has been linked to neurodegeneration ${ }^{16}$. Converging evidence suggests 
that impaired glucose metabolism may contribute to cognitive dysfunction seen in $\mathrm{AD}$. In vivo, people with AD demonstrated consistent and progressive cerebral metabolic rate for glucose reductions when imaged with positron emission tomography ${ }^{45}$. Hypometabolism of glucose contributes to the deposition of amyloid and beta tau proteins in the development of AD. The hippocampus in particular is vulnerable to glucose insufficiency, and in AD cerebral glucose metabolism is reduced by $20-40 \%{ }^{46}$. The KD offers a bypass of glucose metabolism by producing ketone bodies utilizable in these regions affected by amyloid beta deposits and neurofibrillary tangles ${ }^{47}$. By providing ketone bodies as an energy source, cognitive function may potentially improve through this mechanism of an alternative fuel.

Oral administration of exogenous ketones may also be a potential therapeutic option for AD. Reger et al. ${ }^{48}$ conducted a double-blind placebo-controlled study assessing the effect of beta-hydroxybutyrate (BHB) on cognition in memory-impaired adults. Twenty individuals with probable AD or amnestic mild cognitive impairment consumed an oral dose of medium chain triglycerides that significantly elevated levels of the ketone body, BHB. Higher ketone levels were associated with greater improvements in paragraph recall ${ }^{48}$ indicating the potential use of medium chain triglycerides as a therapeutic strategy for cognitive impairment.

Henderson et al. ${ }^{49}$ evaluated the effects of an oral ketogenic compound, AC-1202, on cognitive performance in individuals with probable AD. This was a 90 day, randomized, double-blind, placebo-controlled, parallelgroup study on 152 individuals. AC-1202 significantly elevated BHB levels 2 hours after administration compared to placebo, which correlated with significant improvements in cognitive abilities in memory, language, orientation, and praxis, as measured by the AD Assessment Scale-Cognitive subscale scores, compared to the placebo group ${ }^{41}$. These results hint at a cognitive advantage in provision of ketone bodies as an alternative energy source in individuals with AD.

Krikorian et al $^{50}$ evaluated the effects of a very low carbohydrate diet in adults diagnosed with mild cognitive impairment. The 23 adults included in the study were assigned to either a very low carbohydrate diet, designed to induce an adapted response to ketosis, or a high carbohydrate diet for 6 weeks. Subjects in the low carbohydrate group demonstrated an improvement in memory performance that correlated with the level of ketosis. The study concluded that consuming very low amounts of carbohydrates even in the short term may improve memory function in older adults with the risk of $\mathrm{AD}$ since ketone levels were positively correlated with memory performance ${ }^{50}$.

\section{Autism Spectrum Disorder}

The prevalence of ASD is significantly higher in people with DS compared to the typical population ${ }^{51}$. Studies report ASD to have an approximate prevalence of $10-18 \%$ in individuals with $\mathrm{DS}^{52,53}$, compared to $1 \%$ in individuals without $\mathrm{DS}^{54}$. Individuals with DS and ASD experience poor social orientating, poor integration of verbal and nonverbal behaviors, and limitations in expressive language and adaptive behavior ${ }^{55}$.

Studies have looked into the $\mathrm{KD}$ as a potential therapeutic option in children with ASD. Evangeliou et al. ${ }^{20}$ conducted a pilot prospective follow-up study analyzing the role of the KD in 30 children with ASD aged 4-10 years for 6 months. They reported improvements in the Childhood Autism Rating Scale in 18 of the 18 children who adhered to the diet for the duration of the six-month study ${ }^{20}$. This study provided preliminary evidence for the efficacy of the $\mathrm{KD}$ as a potential additional or alternative therapy for ASD. Recently, El-Rashidy et al. ${ }^{19}$ compared the effect of the KD versus a normal diet in a group of 45 autistic children who were 3-8 years of age. The KD group showed significant improvement in Childhood Autism Rating Scale scores. The total Autism Treatment Evaluation Test questionnaire (ATEC) scores were significantly decreased from $41.70 \pm$ 5.52 to $33.70 \pm 4.2$ following implementation of the $\mathrm{KD}$, indicating a decrease in severity of ASD symptoms seen in these children ${ }^{19}$. The subscales in the ATEC that significantly improved included speech/language/communication, sociability, and sensory/cognitive awareness.

Lee et al. ${ }^{21}$ implemented a modified ketogenic glutenfree diet with supplemental medium chain triglycerides in 15 children with ASD aged 2 to 17 years for 3 months. The Autism Diagnostic Observation Schedule, $2^{\text {nd }}$ edition (ADOS-2) and the Childhood Autism Rating Scale-Second Edition (CARS-2) were used to assess the participants. After 3 months, approximately $50 \%$ of the children showed moderate to substantial improvement in ADOS-2 scores. Moreover, $50 \%$ of the participants had significant improvements in CARS-2 scores in the areas of imitation, body use, and fear or nervousness. These results suggest that the KD may be a potential safe and effective treatment that should be considered for treatment of social impairments in children with $\mathrm{ASD}^{21}$. Although there is some evidence for the potential benefits of the KD in ASD, additional studies are warranted.

\section{Cognition and Behavior}

The KD diet has been effective in treating glucose transporter protein 1 deficiency syndrome and pyruvate dehydrogenase complex deficiency. Studies have investigated effects on cognition and behavior after KD implementation in these individuals. Ramm-Peterson et al. ${ }^{56}$ implemented the KD for 6-17 months in 6 patients diagnosed with glucose transporter protein 1 deficiency 
syndrome. Patients in the study had early-onset seizures and developmental delay. All 6 patients demonstrated improvements in general alertness, expressive language, articulation, and physical endurance shortly after starting the $\mathrm{KD}^{56}$. All patients responded to the KD with $>90 \%$ seizure reduction. Movement disorders also improved shortly after dietary intervention. Interestingly, younger patients showed the greatest improvement in cognition particularly with respect to receptive language, expressive verbal language, and cognitive index, yet adults did achieve improvements as well ${ }^{56}$.

Another study by Sofou et al..$^{57}$ implemented the KD on children diagnosed with pyruvate dehydrogenase complex deficiency. Nineteen children initiated the KD at a median age of 2.5 years. Patients were maintained on the KD for a median of 2.9 years. The KD had a positive effect on seizure frequency in all patients with half experiencing seizure freedom within 1 year. Improvements in speech and language were observed in 15 of 19 patients during KD implementation. Behavioral and social functioning were assessed, and all but one patient became more active during treatment, described as being more alert and less somnolen ${ }^{57}$.

Park et al. ${ }^{58}$ conducted a retrospective chart review on 12 children with intractable epilepsy associated with tuberous sclerosis complex who were put on the KD. After 3 months of KD initiation, 10 patients had $>50 \%$ seizure reduction. Moreover, 7 patients exhibited qualitative improvements in cognition and behavior, as reported by caregivers/parents after KD initiation ${ }^{58}$.

The KD is potentially beneficial in the areas of neurobehavioral development, emotional, and social behaviors, and life ability in children with global developmental delay ${ }^{22}$. Zhu et al. ${ }^{22}$ conducted a prospective case-control study for hospitalized children with global developmental delay who were divided into a KD treatment group or a conventional treatment group. Both groups received comprehensive rehabilitation training and were assessed with the Gesell Developmental Scale, Chinese version of the Urban Infant-Toddler Social and Emotional Assessment/Achenbach Child Behavior Checklist, and Infants-Junior High School Students' Social Life Abilities Scale before and after 3, 6, and 9 months of treatment. The KD treatment group had significantly greater improvements in the scores of the adaptive, fine motor, and language quotients of the Gesell Developmental Scale compared with the conventional treatment group at all 3 time points. The KD treatment group also had significantly greater improvements in the Chinese version of Urban InfantToddler Social and Emotional Assessment/Achenbach Child Behavior Checklist scores than the conventional treatment group. After 9 months of treatment the KD treatment group had greater improvements on the Social Life Abilities Scale. The authors concluded that the KD can improve neurobehavioral development and emotional behaviors in children with global developmental delay, with few adverse effects ${ }^{22}$.

\section{Challenges to implementing the Ketogenic Diet in} Down syndrome

There are barriers to implementing the $\mathrm{KD}$ in individuals with DS. The biggest challenge is compliance. The KD can be a challenging diet to implement, particularly on the part of the care providers. This requires careful attention to meal planning and securing the partnership of all involved with the patient including the school staff, friends, and family members. Children with DS often have inadequate nutrition due to problems chewing and swallowing certain foods, ${ }^{2}$ and this needs to be addressed in designing ketogenic friendly meals. Individuals with DS often experience constipation and this could be compounded, since it is an adverse effect of the $\mathrm{KD}^{16}$. In addition, there are challenges with compliance on the part of the individual, particularly in those with restricted food interests, or those that have access to food outside of their prepared meals. The KD can be modified to allow for improved compliance and ease of administration. Working with a physician and dietitian/nutritionist trained in the KD can help to optimize diet compliance and management of side effects. Creating a network of support with other parents and family members can also greatly improve the success of the $\operatorname{diet}^{23}$. A properly formulated KD with careful attention to food choices and proper food textures can provide adequate nutrition in children with DS.

\section{Summary}

DS is a common cause of intellectual disability and involves multiple comorbidities including epilepsy and AD. Studies have shown correlation between early onset dementia, AD-like pathology, and seizure activity with cognitive decline seen in DS. There is a lack of medicinal and dietary therapeutic interventions that improve the quality of life for those with DS. Recently, the KD has been studied for its potential to improve cognition and behavior in individuals with epilepsy and ASD. The KD is a novel therapeutic option that may be potentially beneficial for individuals with DS and warrants investigation.

\section{Acknowledgements}

Shriners Hospitals for Children ${ }^{\circledR}$ - Honolulu

\section{Funding and Sponsorship}

This research did not receive any specific grant form funding agencies in the public, commercial, or not-forprofit sectors.

\section{Declaration of Interest}

There are no conflicts of interests. 


\section{References}

1. World Health Organization. Genes and Human Disease 2017, USA. Available at: http://www.who.int/genomics/public/geneticdiseases/ en/index1.html. Accessed on July 8, 2017

2. Roizen NJ, Patterson D. Down's syndrome. Lancet. 2003; 361: 1281 89.

3. Roberts JE, Price J, Malkin C. Language and communication development in down syndrome. Developmental Disabilities Research Reviews. 2007; 13: 26-35.

4. Maatta T, Tervo-Maatta T, Taanila A, et al. Mental health, behaviour and intellectual abilities of people with Down syndrome. Down Syndrome Research and Practice. 2006; 11(1): 37-43

5. Annus T, Wilson LR, Hong YT, etal. The pattern of amyloid accumulation in the brains of adults with Down syndrome. Alzheimer's \& dementia: the journal of Alzheimer's Association. 2016; 12(5): 538-545.

6. Schupf N, Sergievsky GH. Genetic and host factors for dementia in Down's syndrome. Br J Psychiatry. 2002; 180: 405-10.

7. Hoyo LD, Xicota L, Sanchez-Benavides G, etal. Semantic verbal fluency pattern, dementia rating scores and adaptive behavior correlate with plasma A $\beta 42$ concentrations in Down syndrome young adults. Front Behav Neurosci. 2015; 18(9): 301.

8. Alexander M, Petri H, Ding Y, Wandel C, Khwaja O, Foskett N. Morbidity and medication in a large population of individuals with Down syndrome compared to the general population. Dev Med child Neurol. 2016; 58: 246-254.

9. McCarron M, McCallion P, Reilly E, et al. A prospective 14-year longitudinal follow-up of dementia in persons with Down syndrome. J Intellect Disabil Res. 2014; 58: 61-70.

10. Lott IT, Doran E, Nguyen VQ et al. Down syndrome and dementia: seizures and cognitive decline. J Alzheimers Dis. 2012; 29(1): 177 185.

11. Puri BK, Ho KW, Singh I. Age of seizure onset in adults with Down's syndrome. Int J Clin Pract. 2001; 55: 442-44.

12. Hughes JR. Absence seizures: a review of recent reports with new concepts. Epilepsy Behav. 2009; 15: 404-12.

13. Livingstone N, Hanratty J, McShane R, et al. Pharmacological interventions for cognitive decline in people with Down syndrome. Cochrane Database of Systematic Reviews. 2015; 10: 1-68.

14. Ellis JM, Tan HK, Gilbert RE, et al. Supplementation with antioxidants and folinic acid for children with Down's syndrome: randomised controlled trial. BMJ. 2008; 336(7644): 594-7.

15. Mazurek D, Wyka J. Down syndrome-genetic and nutritional aspects of accompanying disorders. Mental Retardation. 2015; 66: 189-94.

16. Boison D, D’Agostino DP, Kossoff EH, et al. Ketogenic diet and metabolic therapies. Masino SA, ed. Oxfaord; New York: Oxford University Press; 2017: 1-425

17. Pulsifer MG, Gordon JM, Brandt J, et al. Effects of ketogenic diet on development and behavior: preliminary report of a prospective study Developmental Medicine and Child Neurology. 2001; 43: 301-306.

18. Ijff DM, Postulart D, Lambrechts D, et al. Cognitive and behavioral impact of the ketogenic diet in children and adolescents with refractory epilepsy: A randomized controlled trial. Epilepsy and Behavior. 2016; 60: 153-157.

19. El-Rashidy O, El-Baz F, El-Gendy, et al. Ketogenic diet versus gluten free casein free diet in autistic children: a case-control study. Metab Brain Dis. 2017: 1-7.

20. Evangeliou A, Vlachonikolis I, Mihailidou H, et al. Application of a ketogenic diet in children with autistic behavior: pilot study. J child Neurol. 2003; 18(2): 113-8.
21. Lee RWY, Corley MJ, Pang A, et al. A modified ketogenic gluten-free diet with MCT improves behavior in children with autism spectrum disorder. Physiology \& Behavior. 2018; 188: 205-211.

22. Zhu DN, Li P, Wang J, \et al. Prospective study of ketogenic diet in treatment of children with global developmental delay. Zhongguo Dang Dai Er Ke Zhi. 2017; 19(10): 1038-1043.

23. Kossoff EH, Turner Z, Doerrer S, et al. The Ketogenic and Modified Atkins Diets: Treatments for epilepsy and other disorders. New York, NY: Demos Medical Publishing; 2016.

24. Kossoff EH, Wang HS. Dietary therapies for epilepsy. Biomed J. 2013; 36: 2-8.

25. Lambrechts DA, de Kinderen RJ, Vles JS, et al. A randomized controlled trial of the ketogenic diet in refractory childhood epilepsy. Acta Neurol Scand. 2016; 135: 231-239.

26. Sharma S, Sankhyan N, Gulati S, et al. Use of the modified Atkins diet for treatment of refractory childhood epilepsy: a randomized controlled trial. Epilepsia. 2013; 54: 481-486.

27. Freeman JM, Vining EP, Pillas DJ, et al. The efficacy of the ketogenic diet-1998: a prospective evaluation of intervention in 150 children. Pediatrics. 1998; 102: 1358-1363.

28. Stafstrom CE, Rho JM. The ketogenic diet as a treatment paradigm for diverse neurological disorders. Front Pharmacol. 2012; 3: 59.

29. Paoli A, Rubini A, Volek JS, et al. Beyond weight loss: a review of the therapeutic uses of very-low-carbohydrate (ketogenic) diets. Eur J Clin Nutr. 2013; 67: 789-96.

30. Simeone TA, Simeone KA, Stafstrom CE, et al. Do ketone bodies mediate the anti-seizure effects of the ketogenic diet? Neuropharmacology. 2018; 133: 233-241.

31. Grocott OR, Herrington KS, Pfeifer $\mathrm{HH}$, et al. Low glycemic index treatment for seizure control in Angelman syndrome: A case series from the center of dietary therapy of epilepsy at the Massachusetts General Hospital. Epilepsy \& Behavior. 2017; 68: 45-50.

32. Lee RY, Wong M. (1999, July 15). Ketogenic diet in the treatment of epilepsy. Retrieved from http://www.medlink.com/article/ ketogenic_diet_in_the_treatment_of_epilepsy

33. Boison D. New insights into the mechanisms of the ketogenic diet. Current Opinion in Neurology. 2017; 30: 187-192.

34. Giordano C, Marchio M, Timofeeva E, et al. Meuroactive peptides as putative mediators of antiepileptic ketogenic diets. Frontiers in Neurology. 2014; 5: 63.

35. Lucchi C, Costa AM, Giordano C, et al. Involvement of PPAR $\gamma$ in the anticonvulsant activity of EP-80317, a ghrelin receptor antagonist. Frontiers in Pharmacology. 2017; 8: 676.

36. Simeone TA, Matthews SA, Samson KK, et al. Regulation of brain PPARgamma2 contributes to ketogenic diet anti-seizure efficacy. Experimental Neurology. 2017; 287: 54-64.

37. Arya R, Kabra M, Gulati S. Epilepsy in children with Down syndrome. Epileptic Disord. 2011; 13(1): 1-7.

38. Czochanska J, Langer-Tyszka B, Losiowski Z, et al. Children who develop epilepsy in the first year of life: a prospective study. Developmental Medicine \& Child Neurology. 1994; 36: 183-92.

39. Hoare P. The development of psychiatric disorders among children with epilepsy. Developmental Medicine \& Child Neurology. 1984; 26: 14-9.

40. Neal EG, Chaffe H, Schwartz RH, et al. The ketogenic diet for the treatment of childhood epilepsy: a randomized controlled trial. The Lancet Neurology. 2008; 7: 500-506.

41. Vining EPG, Freeman JM, Ballaban-Gil K, et al. A multicenter study of the efficacy of the ketogenic diet. Archives of Neurology. 1998; 55: 1433-7. 
42. Hallbook T, Lundgren J, Rosen I. Ketogenic diet improves sleep quality in children with therapy-resistant epilepsy. Epilepsia. 2007; 48(1) 59-65.

43. Lucchi C, Marchio M, Carmaschi E, et al. Electrographic changes accompanying recurrent seizures under Ketogenic diet treatment. Pharmaceuticals. 2017; 10: E82.

44. Head E, Powell D, Gold BT, et al. Alzheimer's disease in Down syndrome. European journal of neurodegenerative disease. 2012; 1(3): 353-364.

45. Reiman EM, Chen K, Alexander GE, et al. Functional brain abnormalities in young adults at genetic risk for late-onset Alzheimer's dementia. Proceedings of the National Academy of Sciences. 2004; 101: 284 289.

46. Hoyer S. Oxidative energy metabolism in Alzheimer brains. Studies in early-onset and late onset cases. Mol Chem Neuropathol 1992; 16 207-24.

47. Castellano CA, Nugent $\mathrm{S}$, Paquet $\mathrm{N}$, et al. Lower brain 18F-Fluorodeoxyglucose uptake but normal 11C-acetoacetate metabolism in mild Alzheimer's disease dementia. J Alzheimers Dis. 2015; 43: 1343-53.

48. Reger MA, Henderson ST, Hale C, et al. Effects of $\beta$-hydroxybutyrate on cognition in memory-impaired adults. Neurobiology of Aging. 2004; 25: 311-314.

49. Henderson ST, Vogel JL, Barr LJ, et al. Study of the ketogenic agent AC1202 in mild to moderate Alzheimer's disease: a randomized, doubleblind, placebo-controlled, multicenter trial. Nutrition \& Metabolism. 2009; 6(31): 1-25.

50. Krikorian R, Shidler MD, Dangelo K, et al. Dietary ketosis enhances memory in mild cognitive impairment. Neurobiol Aging. 2012; 33 : e19-27.

51. Moss J, Richards C, Nelson L, et al. Prevalence of autism spectrum disorder symptomatology and related behavioral characteristics in individuals with Down syndrome. Autism. 2013; 17(4): 390-404.

52. Carter JC, Capone GT, Gray RM, et al. Autistic-spectrum disorders in Down syndrome: further delineation and distinction from other behavioral abnormalities. Am J Med genet B Neuropsychiatr Genet. 2007; 144B(1): 87-94

53. Hepburn S, Philofsky A, Fidler DJ, et al. Autism symptoms in toddlers with Down syndrome: a descriptive study. J Appl Res Intellect Disabil. 2008; 21(1): 48-57.

54. Christensen DL, Baio J, Braun KVN, et al. Prevalence and characteristics of autism spectrum disorder among children aged 8 years - autism and developmental disabilities monitoring network, 11 sites, United States, 2012. MMWR CDC Surveill Summ. 2016; 65(3): 1-23.

55. Rachubinski AL, Hepburn S, Elias ER, et al. The co-occurrence of Down syndrome and autism spectrum disorder: is it because of additional genetic variations? Prenatal Diagnosis. 2017; 37: 31-36.

56. Ramm-Pettersen A, Stabell KE, Nakken KO, et al. Does ketogenic diet improve cognitive function in patients with GLUT1-DS? A 6- to 17-month follow-up study. Epilepsy \& Behavior. 2014; 39: 111-115.

57. Sofou K, Dahlin M, Hallbook T, et al. Ketogenic diet in pyruvate dehydrogenase complex deficiency: short-and long-term outcomes. J Inherit Metab Dis. 2017; 40: 237-245.

58. Park S, Lee EJ, Eom S, et al. Ketogenic diet for the management of epilepsy associated with tuberous sclerosis complex in children. Journal of Epilepsy Research. 2017; 7: 45-49. 